\title{
Sliding Mode Control for Nonlinear Systems Based on Singular Value Decomposition
}

\author{
Dan Zhao ${ }^{1}$, Hongyu Zhang ${ }^{2}$, Yue $\mathrm{Han}^{3}$ and Zisong Xiao ${ }^{4}$ \\ ${ }^{1}$ Department of Fundamental Teaching, Shenyang Institute of Engineering, \\ Shenyang \\ ${ }^{2}$ State Grid Liaoning Electric Power Supply Co., LTD., Liaoning Province, China \\ ${ }^{3}$ Electric Power Research Institute, State Grid Liaoning Electric Power Supply Co. \\ Ltd, Shenyang, China \\ ${ }^{4}$ Liaoning State Grid Electric Power Transmission \& Transformation Engineering \\ Company \\ "zhaodan@sie.edu.cn
}

\begin{abstract}
The sliding mode control problem is investigated for a class of nonlinear systems with the unmatched nonlinearity which satisfies a quadratic constraint. Based on Lyapunov stability theory, utilizing the singular value decomposition method, the sliding mode control is formulated to stabilize the considered systems. Meanwhile, the bounds on the nonlinear term which this type of systems can tolerate without going unstable are maximized. More importantly, an efficient sliding mode control law with tuned parameters is designed to guarantee the state variables to converge to the reference state in finite time and remain onto it in the subsequent time. Finally, simulation results are presented to illustrate the effectiveness of the control strategy.
\end{abstract}

Keywords: Sliding mode control, unmatched condition, tuned parameters, bound on the nonlinearity

\section{Introduction}

Since sliding mode control (SMC) possesses the strong robustness ability to eliminate or compensate the model uncertainties, parameter variations, and external disturbances [1-5], SMC is an effective control approach and is extensively researched and applied [6-15]. In [6], a novel SMC law is proposed to deliver the tip of a flexible asymmetric-tipped needle to a desired point or to track a desired trajectory within tissue. In [7], an approach known as the TSMC is employed to achieve the objective of fast converging times without excessive control effort. In particular, in [8], the versatility of electric drives and functional goals of control show the effectiveness of the proposed SMC methodology. In [9], using a principle of splitting the compact set of unknown parameters, the authors propose a multiple model control-based SMC technique to reduce the level of parametric uncertainty. In [10], the chattering phenomenon is alleviated by a proposed novel Lyapunovbased, variable-gain super-twisting algorithm. In [11], an active steering assistance system for heavy vehicle is developed and a sliding mode observer estimator is developed to prevent lane departure and let the vehicle follow the road's centre line, and to estimate the vehicle dynamics, respectively. In [12], the authors develop the adaptive control strategy to identify bounded uncertainties for eliminating the requirement of boundaries needed in the conventional design. In [13], a

* Corresponding Author 
methodology combined I \& I adaptive scheme with SMC is employed to allow for prescribed dynamics to be assigned to the estimation error. Also, this proposed method is applied to control a class of power systems to illustrate its effectiveness. In [14], a principle originated from the concept of multidimensional sliding mode is proposed to suppress the chattering effect. An integral sliding surface is proposed for linear time-invariant implicit systems in [15]. And a stabilizing controller is designed to compensate the matched perturbations.

However, there are few reports on optimization for control gain and upper bound of the unmatched nonlinearity, which is the motivation of the present paper. In this paper, two state transformation techniques, which are singular value decomposition transformation and descriptor system model transformation, are employed to obtain the existence condition of sliding surface and the upper bound of the unmatched nonlinear term. Also, as a key characteristic of this paper, the tuned parameters are introduced to reduce the chattering phenomenon. Under the solvable condition of the convex problem, one can obtain the upper bound on the nonlinear term which guarantees the considered systems are stable.

The problem formulation for the considered system based on sliding mode control law is described in Section 2. The sliding surface design and the reaching motion control design are developed in Section 3. In Section 4, a numerical example is given to illustrate the proposed method. Finally, one can obtain some conclusions in Section 5 .

\section{Problem Formulation}

Consider the following nonlinear systems

$$
\begin{aligned}
& \dot{x}(t)=[A+\Delta A(t)] x(t)+\left[A_{\sigma}+\Delta A_{\sigma}(t)\right] x(t-\sigma(t))+B u(t)+h(x) \\
& x(t)=\phi(t), \quad t \in\left[-f_{0}, 0\right]
\end{aligned}
$$

where $x(t) \in \mathfrak{R}^{n} \quad$ is the state of the system, and $u(t) \in \mathfrak{R}^{m}$ is the input vector. $A \in \mathfrak{R}^{n \times n}, A_{\sigma} \in \mathfrak{R}^{n \times n}$, and $B \in \mathfrak{R}^{n \times m}$ are known constant matrices, and $h: \mathfrak{R}^{n} \rightarrow \mathfrak{R}^{n}$ represents a piecewise-continuous nonlinear function satisfying $h(0)=0$. It is assumed that the nonlinear term $h(x)$ can be bounded by a quadratic inequality

$$
h^{\mathrm{T}}(x) h(x) \leq \alpha^{2} x^{\mathrm{T}} H^{\mathrm{T}} H x
$$

where $H$ is a constant matrix, and $\alpha>0$ is a scalar parameter. $\phi(t)$ is the initial condition. $\sigma(t)$ is the time-varying delays. Assume that there exist constants $f_{0}$, and $f$ satisfying

$$
0 \leq \sigma(t) \leq f_{0}, \quad \dot{\sigma}(t) \leq f<1 .
$$

Time-varying parametric uncertainties $\Delta A(t)$ and $\Delta A_{\sigma}(t)$ are assumed to be of the following form

$$
\Delta A(t)=E F(t) D, \quad \Delta A_{\sigma}(t)=E_{\sigma} F_{\sigma}(t) D_{\sigma}
$$

where $E, D, E_{\sigma}$ and $D_{\sigma}$ are constant matrices of appropriate dimensions, and $F(t)$ and $F_{\sigma}(t)$ are the unknown matrix function satisfying

$$
F^{\mathrm{T}}(t) F(t) \leq I, F_{\sigma}^{\mathrm{T}}(t) F_{\sigma}(t) \leq I, \forall t \geq 0 .
$$


In this paper, we assume that $\operatorname{rank}(B)=m$. One can easily get the singular value decomposition of $B$ :

$$
B=\left[\begin{array}{ll}
U_{1} & U_{2}
\end{array}\right]\left[\begin{array}{c}
\Sigma \\
0_{(n-m) \times m}
\end{array}\right] V^{\mathrm{T}}
$$

where $\Sigma \in \mathfrak{R}^{m \times m}$ is a diagonal positive-definite matrix, $U_{1} \in \mathfrak{R}^{n \times m}, U_{2} \in \mathfrak{R}^{n \times(n-m)}$ and $V \in \mathfrak{R}^{m \times m}$ are unitary matrices.

Choose $z=\Gamma x$, where $\Gamma^{T}=\left[\begin{array}{ll}U_{1} & U_{2}\end{array}\right]$, we can obtain another form of (1) as follows:

$$
\begin{aligned}
\dot{z}_{1}(t) & =\left[\bar{A}_{11}+\Delta \bar{A}_{11}(t)\right] z_{1}(t)+\left[\bar{A}_{12}+\Delta \bar{A}_{12}(t)\right] z_{2}(t)+\left[\bar{A}_{\sigma 11}+\Delta \bar{A}_{\sigma 11}(t)\right] z_{1}(t-\sigma(t)) \\
& +\left[\bar{A}_{\sigma 12}+\Delta \bar{A}_{\sigma 12}(t)\right] z_{2}(t-\sigma(t))+B_{1} u(t)+\bar{h}_{1}\left(\Gamma^{-1} z\right) \\
\dot{z}_{2}(t) & =\left[\bar{A}_{21}+\Delta \bar{A}_{21}(t)\right] z_{1}(t)+\left[\bar{A}_{22}+\Delta \bar{A}_{22}(t)\right] z_{2}(t)+\left[\bar{A}_{\sigma 21}+\Delta \bar{A}_{\sigma 21}(t)\right] z_{1}(t-\sigma(t)) \\
& +\left[\bar{A}_{\sigma 22}+\Delta \bar{A}_{\sigma 22}(t)\right] z_{2}(t-\sigma(t))+\bar{h}_{2}\left(\Gamma^{-1} z\right) \\
z_{1}(t) & =\bar{\phi}_{1}(t), \quad t \in\left[-f_{0}, 0\right] \\
z_{2}(t) & =\bar{\phi}_{2}(t), \quad t \in\left[-f_{0}, 0\right]
\end{aligned}
$$

where $z_{1}(t) \in \mathfrak{R}^{m}, z_{2}(t) \in \mathfrak{R}^{n-m}, \bar{A}_{11}=U_{1}^{\mathrm{T}} A U_{1}, \bar{A}_{12}=U_{1}^{\mathrm{T}} A U_{2}, \bar{A}_{21}=U_{2}^{\mathrm{T}} A U_{1}$, $\bar{A}_{22}=U_{2}^{\mathrm{T}} A U_{2}, \bar{h}_{1}\left(\Gamma^{-1} z\right)=U_{1}^{\mathrm{T}} h\left(\Gamma^{-1} z\right), \bar{h}_{2}\left(\Gamma^{-1} z\right)=U_{2}^{\mathrm{T}} h\left(\Gamma^{-1} z\right)$.

We construct a sliding surface as follows:

$$
S(t)=\left[\begin{array}{ll}
I & C
\end{array}\right] z(t)=z_{1}(t)+C z_{2}(t)=0
$$

Noting the second equation of (6) and (7), we can obtain the following sliding motion:

$$
\begin{aligned}
\dot{z}_{2}(t) & =\left[\bar{A}_{22}-\bar{A}_{21} C+\Delta \bar{A}_{22}(t)+\Delta \bar{A}_{21}(t) C\right] z_{2}(t)+\bar{h}_{2}\left(\Gamma^{-1} z\right) \\
& +\left[\bar{A}_{\sigma 22}-\bar{A}_{\sigma 21} C+\Delta \bar{A}_{\sigma 22}(t)+\Delta \bar{A}_{\sigma 21}(t) C\right] z_{2}(t-\sigma(t)) \\
z_{2}(t) & =\bar{\phi}_{2}(t), \quad t \in\left[-f_{0}, 0\right]
\end{aligned}
$$

where $\bar{h}_{2}^{\mathrm{T}}\left(\Gamma^{-1} z\right) \bar{h}_{2}\left(\Gamma^{-1} z\right) \leq \alpha^{2} z_{2}^{\mathrm{T}}(t)\left[\begin{array}{ll}-C^{\mathrm{T}} & I\end{array}\right] \bar{H}^{\mathrm{T}} \bar{H}\left[\begin{array}{c}-C \\ I\end{array}\right] z_{2}(t)$, and $\bar{H}=H \Gamma^{-1}$.

In this paper, our objective is to design a sliding surface $S(t)$ and a reaching motion control law $u(t)$ satisfying the following two conditions:

(a) Sliding motion (8) is quadratically stable with upper bound $\alpha^{*}$, that is,

for any given $\bar{\alpha}$, find out the upper bound $\alpha^{*}$, so that for all $\alpha$ satisfying $\bar{\alpha}<\alpha<\alpha^{*}$, sliding motion (8) is quadratically stable;

(b) Find an upper bound $\alpha^{*}$, such that (6) is asymptotically stable under the reaching control law $u(t)$.

Lemma 1 For any constant matrix $M \in \mathfrak{R}^{q \times l}$, inequality

$$
2 u^{\mathrm{T}} M v \leq r u^{\mathrm{T}} M G M^{\mathrm{T}} u+\frac{1}{r} v^{\mathrm{T}} G^{-1} v, \quad u \in \mathfrak{R}^{q}, v \in \mathfrak{R}^{l}
$$


holds for any pair of symmetric positive definite matrix $G \in \mathfrak{R}^{l \times l}$ and positive number $r>0$.

\section{Main Result}

Theorem 1 The sliding motion (8) is quadratically stable with upper bound $\alpha^{*}$, if the following optimization problem

$$
\min \gamma+K_{X}+K_{Q_{2}}
$$

subject to

$$
\begin{aligned}
\Omega=\left[\begin{array}{cccccccc}
\Omega_{11} & \Omega_{12} & \Omega_{13} & \Omega_{14} & \Omega_{15} & \Omega_{16} & 0 & \Omega_{18} \\
* & \Omega_{22} & \Omega_{23} & \Omega_{24} & 0 & \Omega_{26} & 0 & 0 \\
* & * & \Omega_{33} & 0 & 0 & 0 & \Omega_{37} & 0 \\
* & * & * & \Omega_{44} & 0 & 0 & 0 & 0 \\
* & * & * & * & \Omega_{55} & 0 & 0 & 0 \\
* & * & * & * & * & \Omega_{66} & 0 & 0 \\
* & * & * & * & * & * & \Omega_{77} & 0 \\
* & * & * & * & * & * & * & \Omega_{88}
\end{array}\right]<0 \\
\\
\gamma-\frac{1}{\bar{\alpha}^{2}}<0 \\
\\
{\left[\begin{array}{cc}
-K_{X} I & X^{\mathrm{T}} \\
* & -I
\end{array}\right]<0 } \\
{\left[\begin{array}{cc}
Q_{2} & I \\
* & K_{Q_{2}} I
\end{array}\right]>0 }
\end{aligned}
$$

has a solution set ( positive scalars $\gamma, \varepsilon_{1}, \varepsilon_{2}, K_{Q_{2}}, K_{X}$, positive definite matrices $Q_{1}, Q_{2}, Q_{3}$, and matrix $X$ ). Furthermore, the gain $C$ of the sliding surface (7) and the upper bound $\alpha^{*}$ are obtained as follows

$$
C=X Q_{2}^{-1}, \quad \alpha^{*}=\sqrt{\frac{1}{\gamma}}
$$

where $\bar{\alpha}$ is a given scalar parameter in (2), and

$$
\begin{gathered}
\Omega_{11}=\bar{A}_{22} Q_{2}-\bar{A}_{21} X+Q_{2} \bar{A}_{22}^{\mathrm{T}}-X^{\mathrm{T}} \bar{A}_{21}^{\mathrm{T}}+Q_{3}, \Omega_{12}=Q_{1}-Q_{2}+Q_{2} \bar{A}_{22}^{\mathrm{T}}-X^{\mathrm{T}} \bar{A}_{21}^{\mathrm{T}}, \\
\Omega_{13}=\bar{A}_{\sigma 22} Q_{2}-\bar{A}_{\sigma 21} X, \quad \Omega_{14}=\varepsilon_{1} U_{2}^{\mathrm{T}} E, \quad \Omega_{15}=Q_{2} U_{2}^{\mathrm{T}} D^{\mathrm{T}}-X^{\mathrm{T}} U_{1}^{\mathrm{T}} D^{\mathrm{T}}, \quad \Omega_{16}=\varepsilon_{2} U_{2}^{\mathrm{T}} E_{\sigma}, \\
\Omega_{18}=\left[\begin{array}{ll}
-X^{\mathrm{T}} & Q_{2}
\end{array}\right] \bar{H}^{\mathrm{T}}, \Omega_{22}=-Q_{2}-Q_{2}+I, \quad \Omega_{23}=\Omega_{13}, \quad \Omega_{24}=\Omega_{14}, \quad \Omega_{26}=\Omega_{16},
\end{gathered}
$$




$$
\begin{gathered}
\Omega_{33}=-(1-f) Q_{3}, \quad \Omega_{37}=Q_{2} U_{2}^{\mathrm{T}} D_{\sigma}^{\mathrm{T}}-X^{\mathrm{T}} U_{1}^{\mathrm{T}} D_{\sigma}^{\mathrm{T}}, \Omega_{44}=-\varepsilon_{1} I, \\
\Omega_{55}=-\varepsilon_{1} I, \quad \Omega_{66}=-\varepsilon_{2} I, \\
\Omega_{77}=-\varepsilon_{2} I, \quad \Omega_{88}=-\gamma I .
\end{gathered}
$$

Proof Choose $P_{2}=Q_{2}^{-1}, P_{1}=Q_{2}^{-1} Q_{1} Q_{2}^{-1}, P_{3}=Q_{2}^{-1} Q_{3} Q_{2}^{-1}$, and construct a Lyapunov functional

$$
V\left(z_{2}(t), y(t), t\right)=\left[\begin{array}{ll}
z_{2}^{\mathrm{T}}(t) & y^{\mathrm{T}}(t)
\end{array}\right]\left[\begin{array}{ll}
I & 0 \\
0 & 0
\end{array}\right]\left[\begin{array}{cc}
P_{1} & 0 \\
P_{2} & P_{2}
\end{array}\right]\left[\begin{array}{c}
z_{2}(t) \\
y(t)
\end{array}\right]+\int_{t-\sigma(t)}^{t} z_{2}^{\mathrm{T}}(s) P_{3} z_{2}(s) d s
$$

Obviously, $V\left(z_{2}(t), y(t), t\right)>0$ for all $\left[z_{2}^{\mathrm{T}}(t) y^{\mathrm{T}}(t)\right] \neq 0$. Applying (3), (4), Lemma1, and the decomposition techniques of matrix to the derivative of $V\left(z_{2}(t), y(t), t\right)$ along the trajectories of the equivalent descriptor system of (8), yield

$$
\dot{V}\left(z_{2}(t), y(t), t\right) \leq \zeta^{\mathrm{T}} \mathrm{Y} \zeta
$$

where $\zeta^{\mathrm{T}}=\left[\begin{array}{lll}z_{2}^{\mathrm{T}}(t) & y^{\mathrm{T}}(t) \quad z_{2}^{\mathrm{T}}(t-\sigma(t)\end{array}\right]$

$$
\begin{aligned}
& \mathrm{Y}=\left[\begin{array}{ccc}
\mathrm{Y}_{11} & \mathrm{Y}_{12} & \mathrm{Y}_{13} \\
* & \mathrm{Y}_{22} & \mathrm{Y}_{23} \\
* & * & \mathrm{Y}_{33}
\end{array}\right]+\left[\begin{array}{c}
U_{2}^{\mathrm{T}} D^{\mathrm{T}}-C^{\mathrm{T}} U_{1}^{\mathrm{T}} D^{\mathrm{T}} \\
0 \\
0
\end{array}\right] F^{\mathrm{T}}(t)\left[E^{\mathrm{T}} U_{2} P_{2} \quad E^{\mathrm{T}} U_{2} P_{2} \quad 0\right] \\
& +\left[\begin{array}{c}
P_{2}^{\mathrm{T}} U_{2}^{\mathrm{T}} E_{\sigma} \\
P_{2}^{\mathrm{T}} U_{2}^{\mathrm{T}} E_{\sigma} \\
0
\end{array}\right] F_{\sigma}(t)\left[\begin{array}{lll}
0 & 0 & D_{\sigma} U_{2}-D_{\sigma} U_{1} C
\end{array}\right]+\left[\begin{array}{c}
P_{2}^{\mathrm{T}} U_{2}^{\mathrm{T}} E \\
P_{2}^{\mathrm{T}} U_{2}^{\mathrm{T}} E \\
0
\end{array}\right] F(t)\left[D U_{2}-D U_{1} C \quad 0 \quad 0\right] \\
& +\left[\begin{array}{c}
0 \\
0 \\
U_{2}^{\mathrm{T}} D_{\sigma}^{\mathrm{T}}-C^{\mathrm{T}} U_{1}^{\mathrm{T}} D_{\sigma}^{\mathrm{T}}
\end{array}\right] F_{\sigma}^{\mathrm{T}}(t)\left[\begin{array}{lll}
E_{\sigma}^{\mathrm{T}} U_{2} P_{2} & E_{\sigma}^{\mathrm{T}} U_{2} P_{2} & 0
\end{array}\right], \\
& \mathrm{Y}_{11}=P_{2}\left(\bar{A}_{22}-\bar{A}_{21} C\right)+\left(\bar{A}_{22}-\bar{A}_{21} C\right)^{\mathrm{T}} P_{2}+P_{3}+\frac{1}{\gamma}\left[\begin{array}{ll}
-C^{\mathrm{T}} & I
\end{array}\right] \bar{H}^{\mathrm{T}} \bar{H}\left[\begin{array}{c}
-C \\
I
\end{array}\right], \\
& \mathrm{Y}_{12}=P_{1}-P_{2}+\left(\bar{A}_{22}-\bar{A}_{21} C\right)^{\mathrm{T}} P_{2}, \quad \mathrm{Y}_{13}=P_{2}\left(\bar{A}_{\sigma 22}-\bar{A}_{\sigma 21} C\right), \quad \mathrm{Y}_{22}=-P_{2}-P_{2}+P_{2} P_{2} \text {, } \\
& \mathrm{Y}_{23}=\mathrm{Y}_{13}, \quad \mathrm{Y}_{33}=-(1-f) P_{3}, \quad \gamma=\frac{1}{\alpha^{2}} \text {. }
\end{aligned}
$$

Pre- and post-multiplying $\mathrm{Y}$ in (15) by $\Theta^{\mathrm{T}}$ and $\Theta$, where $\Theta^{\mathrm{T}}=\operatorname{diag}\left\{P_{2}^{-1}, P_{2}^{-1}, P_{2}^{-1}, P_{2}^{-1}\right\}$, thus one can obtain the following matrix: 


$$
\begin{aligned}
& \overline{\mathrm{Y}}=\left[\begin{array}{ccc}
\overline{\mathrm{Y}}_{11} & \overline{\mathrm{Y}}_{12} & \overline{\mathrm{Y}}_{13} \\
* & \overline{\mathrm{Y}}_{22} & \overline{\mathrm{Y}}_{23} \\
* & * & \overline{\mathrm{Y}}_{33}
\end{array}\right]+\left[\begin{array}{c}
Q_{2} U_{2}^{\mathrm{T}} D^{\mathrm{T}}-X^{\mathrm{T}} U_{1}^{\mathrm{T}} D^{\mathrm{T}} \\
0 \\
0
\end{array}\right] F^{\mathrm{T}}(t)\left[\begin{array}{lll}
E^{\mathrm{T}} U_{2} & E^{\mathrm{T}} U_{2} & 0
\end{array}\right]
\end{aligned}
$$

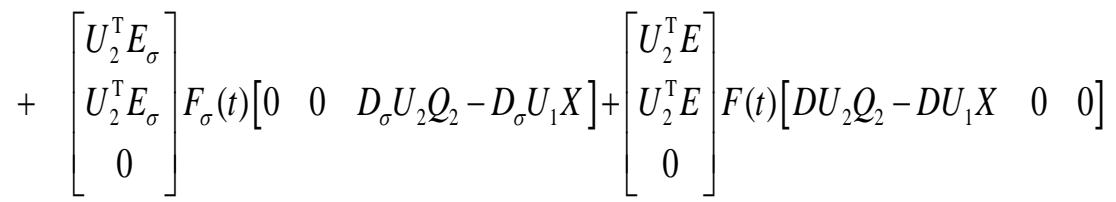

$$
\begin{aligned}
& +\left[\begin{array}{c}
0 \\
0 \\
Q_{2} U_{2}^{\mathrm{T}} D_{\sigma}^{\mathrm{T}}-X^{\mathrm{T}} U_{1}^{\mathrm{T}} D_{\sigma}^{\mathrm{T}}
\end{array}\right] F_{\sigma}^{\mathrm{T}}(t)\left[\begin{array}{lll}
E_{\sigma}^{\mathrm{T}} U_{2} & E_{\sigma}^{\mathrm{T}} U_{2} & 0
\end{array}\right], \\
& \text { where } \overline{\mathrm{Y}}_{11}=\bar{A}_{22} Q_{2}-\bar{A}_{21} X+Q_{2} \bar{A}_{22}-X^{\mathrm{T}} \bar{A}_{21}^{\mathrm{T}}+Q_{3}+\frac{1}{\gamma}\left[\begin{array}{ll}
-X^{\mathrm{T}} & Q_{2}
\end{array}\right] \bar{H}^{\mathrm{T}} \bar{H}\left[\begin{array}{c}
-X \\
Q_{2}
\end{array}\right] \text {, } \\
& \overline{\mathrm{Y}}_{12}=Q_{1}-Q_{2}+Q_{2} \bar{A}_{22}^{\mathrm{T}}-X^{\mathrm{T}} \bar{A}_{21}^{\mathrm{T}}, \quad \overline{\mathrm{Y}}_{13}=\bar{A}_{\sigma 22} Q_{2}-\bar{A}_{\sigma 21} X, \quad \overline{\mathrm{Y}}_{22}=-Q_{2}-Q_{2}+I, \\
& \overline{\mathrm{Y}}_{23}=\overline{\mathrm{Y}}_{13}, \quad \overline{\mathrm{Y}}_{33}=-(1-f) Q_{3}, \quad \gamma=\frac{1}{\alpha^{2}} .
\end{aligned}
$$

By Schur complement lemma, the condition $\Omega<0$ in (10) is equivalent to $\bar{Y}<0$ in (16). Obviously, $\overline{\mathrm{Y}}<0$ means $\mathrm{Y}<0$. From the condition $\mathrm{Y}<0$ in (15), there exists a constant $\rho>0$, such that

$$
\dot{V}\left(z_{2}(t), y(t), t\right) \leq-\rho\left\|z_{2}(t)\right\|^{2} .
$$

Hence, by conditions (14\}) and (17), we can conclude that the sliding motion (8) with (3) and (4) is quadratically stable.

Furthermore, for some desired value $\bar{\alpha}$ satisfying (2), the design of gain $C$ can be formulated as an LMI problem in $\gamma, \varepsilon_{1}, \varepsilon_{2}, K_{Q_{2}}, K_{X}, Q_{1}, Q_{2}, Q_{3}, X$ in (9). Also, one can obtain the upper bound $\alpha^{*}$ which guarantees the quadratic stability for the sliding motion (8) with all $\alpha$ satisfying $\bar{\alpha}<\alpha \leq \alpha^{*}$ in (2) if the LMI optimization is feasible. This completes the proof.

The following result is about the reaching motion control design for the considered systems.

Theorem 2 Suppose the optimization problem (9) has solutions: positive scalars $\gamma, \varepsilon_{1}, \varepsilon_{2}, K_{Q_{2}}, K_{X}$, positive definite matrices $Q_{1}, Q_{2}$, and $Q_{3}$, matrix $X$, and the sliding surface is given by (7). Then the trajectory of the closed-loop system (6) can be driven onto the sliding surface in finite time with the control

$$
u(t)=-B_{1}^{-1}\left[K S+\theta \operatorname{sign}(S)+\bar{C} \bar{A} z(t)+\bar{C} \bar{A}_{\sigma} z(t-\sigma(t))+\operatorname{diag}(\operatorname{sign}(S))\left(N_{1}+N_{2}+N_{3}\right)\right]
$$

where

$\operatorname{diag}(\operatorname{sign}(S))=\operatorname{diag}\left\{\operatorname{sign}\left(s_{1}\right), \operatorname{sign}\left(s_{2}\right), \cdots, \operatorname{sign}\left(s_{m}\right)\right\}, K=\operatorname{diag}\left\{k_{1}, k_{2}, \cdots, k_{m}\right\}$,

$\theta=\operatorname{diag}\left\{\theta_{1}, \theta_{2}, \cdots, \theta_{m}\right\}, K_{i}, \theta_{i}$ are positive constants, and 


$$
\begin{gathered}
N_{1}=\left[\begin{array}{c}
N_{11} \\
N_{21} \\
\vdots \\
N_{m 1}
\end{array}\right]=\left[\begin{array}{c}
\left|\bar{c}_{1} \Gamma E D \Gamma^{-1} z(t)\right| \\
\left|\bar{c}_{2} \Gamma E D \Gamma^{-1} z(t)\right| \\
\vdots \\
\left|\bar{c}_{m} \Gamma E D \Gamma^{-1} z(t)\right|
\end{array}\right], N_{2}=\left[\begin{array}{c}
N_{12} \\
N_{22} \\
\vdots \\
N_{m 2}
\end{array}\right]=\left[\begin{array}{c}
\left|\bar{c}_{1} \Gamma E_{\sigma} D_{\sigma} \Gamma^{-1} z(t-\sigma(t))\right| \\
\left|\bar{c}_{2} \Gamma E_{\sigma} D_{\sigma} \Gamma^{-1} z(t-\sigma(t))\right| \\
\vdots \\
\left|\bar{c}_{m} \Gamma E_{\sigma} D_{\sigma} \Gamma^{-1} z(t-\sigma(t))\right|
\end{array}\right], \\
\bar{C}=\left[\begin{array}{ll}
I & C
\end{array}\right]=\left[\begin{array}{c}
\bar{c}_{1} \\
\bar{c}_{2} \\
\vdots \\
\bar{c}_{m}
\end{array}\right], N_{3}=\left[\begin{array}{c}
N_{13} \\
N_{23} \\
\vdots \\
N_{m 3}
\end{array}\right]=\left[\begin{array}{c}
\sqrt{\frac{1}{\gamma}}\left|\bar{c}_{1} \Gamma H \Gamma^{-1} z(t)\right| \\
\sqrt{\frac{1}{\gamma}}\left|\bar{c}_{2} \Gamma H \Gamma^{-1} z(t)\right| \\
\vdots \\
\sqrt{\frac{1}{\gamma}}\left|\bar{c}_{m} \Gamma H \Gamma^{-1} z(t)\right|
\end{array}\right] .
\end{gathered}
$$

Proof From the sliding surface (7), we obtain the following derivative of $S$ :

$$
\dot{S}=\left[\begin{array}{ll}
I & C
\end{array} \dot{z}(t)=\bar{C}[\bar{A}+\Delta \bar{A}(t)] z(t)+\bar{C}\left[\bar{A}_{\sigma}+\Delta \bar{A}_{\sigma}(t)\right] z(t-\sigma(t))+B_{1} u(t)+\bar{C} \bar{h}\left(\Gamma^{-1} z\right)\right.
$$

that is,

$$
\begin{aligned}
\dot{s}_{i}= & -\left[\operatorname{sign}\left(s_{i}\right) N_{i 1}-\bar{c}_{i} \Gamma E F(t) D \Gamma^{-1} z(t)\right]-\left[\operatorname{sign}\left(s_{i}\right) N_{i 3}-\overline{c_{i}} \bar{h}\left(\Gamma^{-1} z\right)\right] \\
& -\left[\operatorname{sign}\left(s_{i}\right) N_{i 2}-\bar{c}_{i} \Gamma E_{\sigma} F_{\sigma}(t) D_{\sigma} \Gamma^{-1} z(t-\sigma(t))\right]-k_{i} s_{i}-\theta_{i} \operatorname{sign}\left(s_{i}\right)
\end{aligned}
$$

Noting that

$$
\bar{c}_{i} \Gamma E F(t) D \Gamma^{-1} z(t) \leq N_{i 1}, \quad c_{i} \Gamma E_{\sigma} F_{\sigma}(t) D_{\sigma} \Gamma^{-1} z(t) \leq N_{i 2}, \bar{c} \bar{h}\left(\Gamma^{-1} z\right) \leq N_{i 3}
$$

thus we have the following conclusion

$$
\begin{array}{ccc}
\dot{s}_{i}<0, & \text { when } \quad s_{i}>0 \\
\dot{s}_{i}>0, & \text { when } \quad s_{i}<0
\end{array}
$$

Hence, the trajectory of system (6) with the control law (18) can be driven onto the sliding surface in finite time and remain there in the subsequent time. This completes the proof.

\section{Numerical Example}

Consider system (1) with following parameters

$$
\begin{gathered}
A=\left[\begin{array}{ccc}
6.3620 & -39.8156 & -18.9435 \\
-9.3447 & -23.1907 & -30.6357 \\
12.1701 & -18.8262 & -38.8607
\end{array}\right], \quad A_{\sigma}=\left[\begin{array}{ccc}
0.3 & 1.8 & -3.6 \\
-2.2 & 2.1 & -1.7 \\
0.5 & 3.4 & 2.3
\end{array}\right], \\
B=\left[\begin{array}{cc}
77.1820 & 75.7316 \\
111.9279 & 115.8190 \\
73.7521 & 69.3812
\end{array}\right], \quad E=\left[\begin{array}{ccc}
-0.2 & -0.3 & -0.02 \\
0.4 & 0.8 & 0.54 \\
0.3 & 0.2 & 0.7
\end{array}\right], \sigma(t)=0.1(2+\sin (t))
\end{gathered}
$$




$$
D=\left[\begin{array}{ccc}
-0.2 & 0.7 & -0.3 \\
0.5 & -0.5 & -0.8 \\
-0.4 & -0.6 & 0.3
\end{array}\right], \quad D_{\sigma}=\left[\begin{array}{ccc}
0.1 & 0.3 & 0.2 \\
-0.1 & -0.1 & 0.1 \\
0.1 & -0.2 & 0.5
\end{array}\right], \quad H=\left[\begin{array}{ccc}
0.3 & -0.3 & -0.1 \\
0.1 & 0.5- & 0.1 \\
0.2 & 0.1 & -0.5
\end{array}\right],
$$

Applying Matlab toolbox to solving the optimization problem (9) subject to (10)-(13), we obtain the following results:

$$
\begin{gathered}
\gamma=5.6093 * 10^{-4}, \quad \varepsilon_{1}=1.3257, \quad \varepsilon_{2}=0.3863, \quad K_{Q_{2}}=0.4876, \quad K_{X}=0.2440, \\
Q_{1}=7.3293, \quad Q_{2}=2.0531, \quad Q_{3}=11.3122, \quad X=\left[\begin{array}{c}
-0.3129 \\
-0.3821
\end{array}\right] .
\end{gathered}
$$

Further computation, we can the following data:

$$
C=X Q_{2}^{-1}=\left[\begin{array}{c}
-0.1524 \\
-0.1861
\end{array}\right], \quad \alpha^{*}=\sqrt{\frac{1}{\gamma}}=42.2227
$$

Under the following initial condition $\quad \phi(0)=\left[\begin{array}{lll}-0.5 & 0.1 & -0.2\end{array}\right]$, when the tuned parameters $K$ and $\theta$ in (18) can be chosen as

$$
K=\left[\begin{array}{rc}
-0.0362 & 0 \\
0 & 13.1584
\end{array}\right], \quad \theta=\left[\begin{array}{cc}
0.001 & 0 \\
0 & 0.002
\end{array}\right] \text {. }
$$

the simulation results are shown in Figure1 -- Figure6 based on the above parameters. From Figure1 and Figure2, we can see that the nonlinear systems (1) without control are divergent. From Figure 3 and Figure4, one can see that the nonlinear system (6) with control are indeed well stabilized. The control signal $u(t)$ of system (6) and the sliding surface (7) are rather smooth in Figure5 and Figure6.

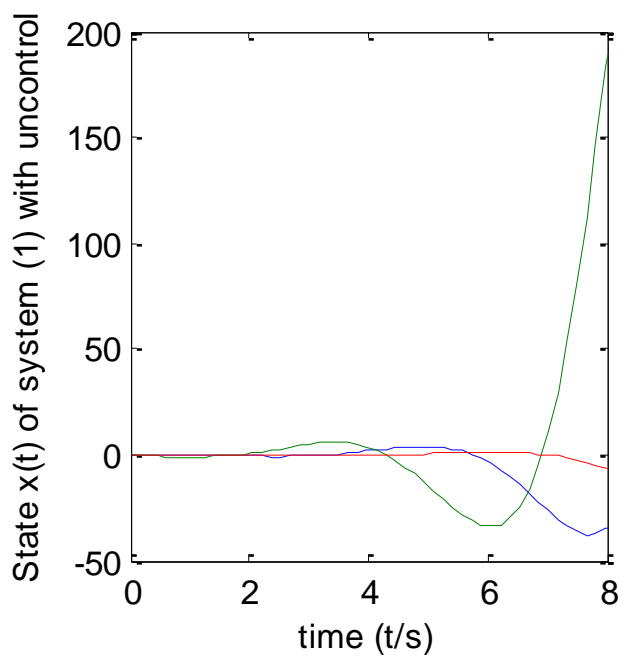

Figure 1. State Response of the Open-Loop System (1) 


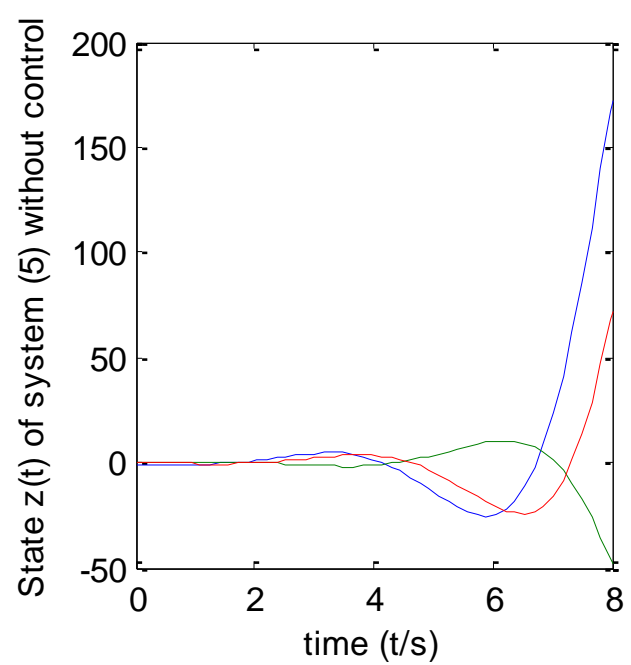

Figure 2. State Response of the Open-Loop System (6)

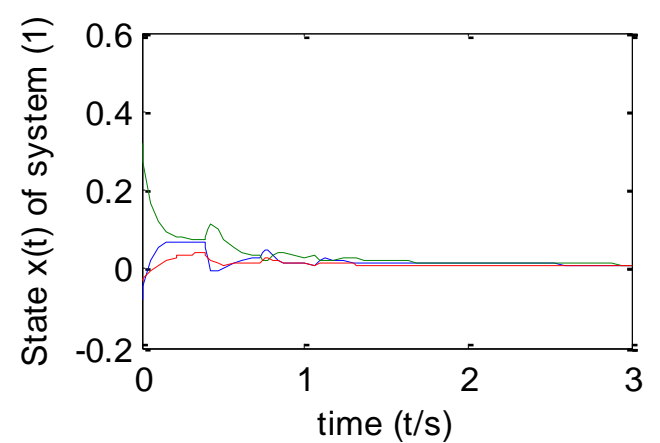

Figure 3. State Response of the Closed-Loop System (1)

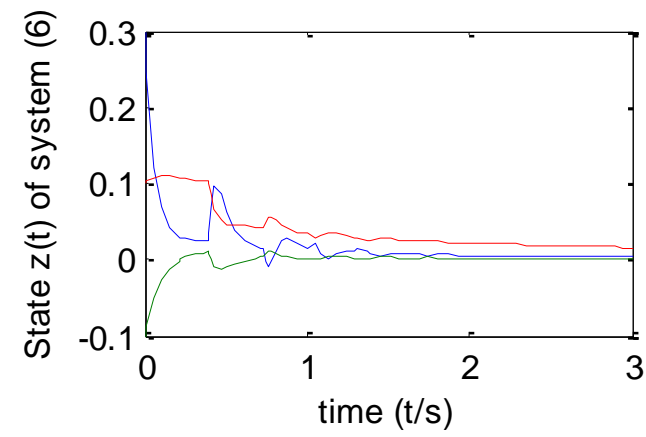

Figure 4. State Response of the Closed-Loop System (6) 


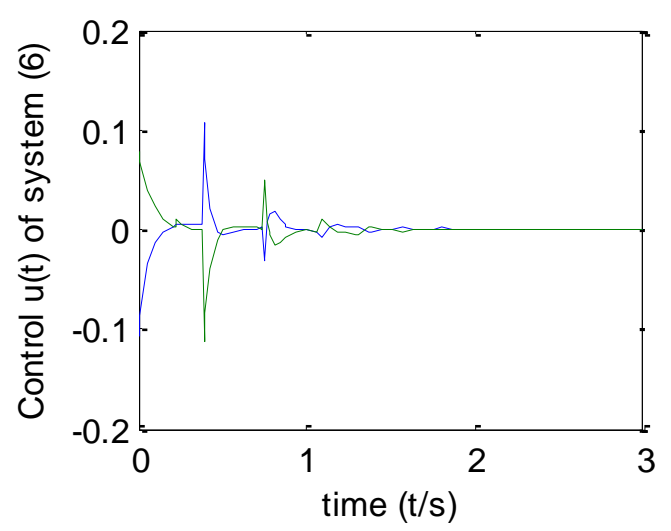

Figure 5. Control Signal of System (6)

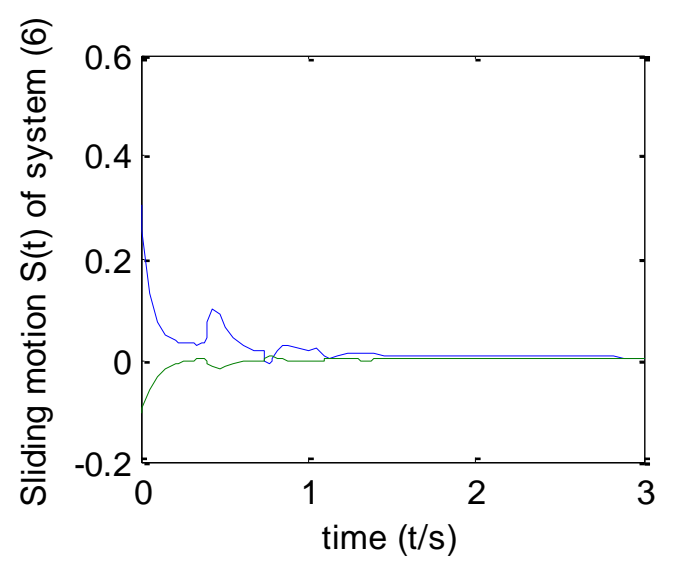

Figure 6. Trajectories of Sliding Variable in Sliding Surface (7)

\section{Conclusion}

The sliding mode control problem of nonlinear systems with unmatched nonlinearity is complex and challenging. Based on $f$ the Lyapunov stability theory, employing the singular- value-decomposition algorithm and descriptor-system-model-transformation method, the sliding surface and the reaching control law are designed, which can be obtained easily by solving the according optimization problem. Also, the upper bound on the nonlinear term which this type of systems can tolerate without going unstable is maximized. The numerical example shows the validity of the proposed algorithm.

\section{Acknowledgements}

This work is supported by National Nature Science Foundation of China under Grant 61304069, 61372195, 61371200, the Fundamental Research Fund of Liaoning Provincial Education Department Key Laboratory under Grant LZ2014050 and the Scientific Research Fund of Liaoning Provincial Education Department under Grant L2012374.

\section{References}

[1] H. Pang and G. Tang, "ICIC Exp. Lett", vol. 4, no. 6, (2010).

[2] Y. Xia, Z. Zhu, and M. Fu, "IET Control Theory Appl", vol. 5, no. 1, (2011).

[3] M. Yan and Y. Shi, "IET Control Theory Appl", vol. 2, no. 8, (2008).

[4] Y. Feng, F. L. Han and X. H. Yu, “Automatica”, vol. 50, no. 4, (2014). 
[5] T. Temal and H. Ashrafiuon, "Electron Lett", vol. 48, no. 15, (2012),

[6] D. C. Rucker, J. Das, H. B. Hunter, P. J. Swaney, M. I. Miga, N. Sarkar and R. J. Webster, "IEEE T Robot", vol. 29, no. 5, (2013).

[7] M. Ghasemi, S. G. Nersesov, G. Clayton and J Franklin I., vol. 351, no. 5, (2014).

[8] V. I. Utkin, "IEEE T IND ELECTRON", vol. 40, no. 1, (1993).

[9] S. Islam and X. P. Liu, "IEEE T Ind Eelectron", vol. 58, no. 6, (2011).

[10] T. Gonzalez, J. A. Moreno and L. Fridman, "IEEE T Automat Cont", vol. 57, no. 8, (2012).

[11] H. Imine and T. Madani, "Int J Robust Nonlin", vol. 23, no. 1, (2013).

[12] W. Q. Tang and Y. L. Cai, "Int J Robust Nonlin", vol. 23, no. 2, (2013).

[13] Z. T. Liu, H. Y. Su and S. W. Pan, "Asian J Control”, vol. 16, no. 1, (2014).

[14] H. Lee, V. Utkin and A. Malinin, "Int J Control", vol. 82, no. 9, (2009).

[15]F. Castanos, D. Hernandez and L. M. Fridman, “Automatica”, vol. 50, no. 3, (2014).

\section{Authors}
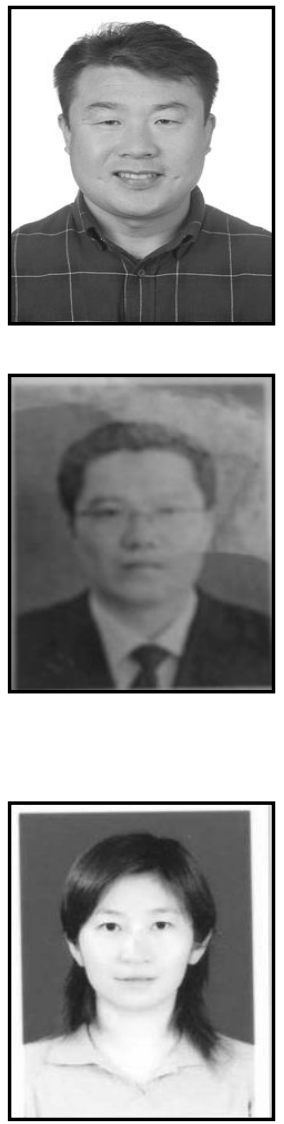

Yue Han, she received the Master degree in Automation of Electrical Power System from North China Electric Power University, Beijing, China, in 2003. She is currently a senior engineer, working in Liaoning Electric Power Research Institute, Shenyang, China. Her current research interests include On-line Monitoring Technology and artificial intelligence. 
International Journal of Control and Automation Vol.8, No.11 (2015) 